Boletín de la Sociedad Geológica Mexicana

VOLUMEN 65, NÚM. 2, 2013, P. 397-404

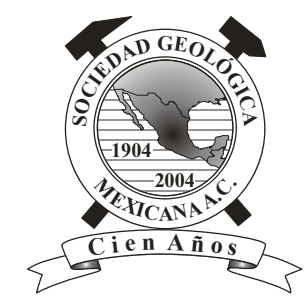

\title{
Revisión estratigráfica y edad de la Formación (Aloformación) Mayrán en el área de Parras, Coahuila, México
}

\author{
Samuel Eguiluz de Antuñano ${ }^{1, *}$, Oscar Carranza-Castañeda ${ }^{2}$ \\ ${ }^{1}$ Geólogo consultor, Circuito Julio Verne 190, Ciudad de Pachuca, Hidalgo, CP 42080, México. \\ ${ }^{2}$ Universidad Nacional Autónoma de México, Campus Juriquilla, Centro de Geociencias, Boulevard Juriquilla 3001, Juriquilla, \\ Querétaro, CP 76230, México. \\ *s_eguiluz@hotmail.com
}

\section{Resumen}

En este artículo se analiza la estratigrafía de la Formación Mayrán y se propone redefinirla como una aloformación, compuesta por una sucesión inferior con estratos de caliza y rocas siliciclásticas, y otra sucesión superior compuesta por capas de tufa y caliza arcillosa, ambas con discordancias producidas por interrupciones en su depósito. Esta aloformación descansa sobre estratos plegados del Grupo Difunta del Cretácico Superior y subyace a una cubierta de suelos del Holoceno. El estrato-tipo de la Aloformación Mayrán se propone en la localidad de Palo Alto y secciones de referencia en las mesas de Cruz Verde y El Durazno, al noreste de la ciudad de Parras de la Fuente, estado de Coahuila. Con base en la estratigrafía, la presencia de molares de Equus y las edades isotópicas de los estratos estudiados, aceptamos una edad de Plioceno a Pleistoceno para estas capas.

Palabras clave: Aloformación, Mayrán, Plioceno, Pleistoceno, tufa, Equus, Coahuila.

\begin{abstract}
This paper discusses the stratigraphy of the Mayrán Formation, and intends to redefine it as an alloformation. The lower part of the alloformation consists of interbedded limestone and sandstone, and the upper succession is tufa and shaly limestone. Both sequences show internal unconformities caused by interruptions during their deposition. The Mayrán alloformation is undeformed and underlie the Upper Cretaceous Difunta Group in angular unconformity, whereas the upper contact is a parallel unconformity (paraconformity) with Holocene soils. The proposed Mayrán Alloformation stratotype is exposed south of the Palo Alto locality, with reference sections in the Cruz Verde and El Durazno plateaus, northeast of Parras de la Fuente, Coahuila state. Based on their stratigraphy, Equus molars, and isotopic ages, these lithostratigraphic units range in age from Pliocene to Pleistocene.
\end{abstract}

Keywords: Mayrán, alloformation, Pliocene, Pleistocene, tufa, Equus, Coahuila. 


\section{Introducción}

La Formación Mayrán aflora en un área de más de $1500 \mathrm{~km}^{2}$. Esta formación se encuentra limitada al sur por las estribaciones del flanco norte de la sierra de Parras y al norte se extiende hasta la proximidad de la población La Paila y la carretera federal 40 , la cual une a las ciudades de Saltillo y Torreón, en el estado de Coahuila. Al oriente su límite llega cercano al poblado de El Tejocote y al poniente la delimita el poblado de San Rafael (Figura 1).

La descripción original de la Formación Mayrán (Imlay, 1936) menciona la presencia de conglomerados bien cementados, con tufas que son cuerpos de roca formados por minerales de carbonato de calcio y precipitados en ambiente lacustre, sin influencia de una fuente termal, cuya etimología proviene de la palabra tufa (de origen chino), dichas tufas se encuentran intercaladas conteniendo moldes de plantas. En esta descripción se menciona la presencia de "huesos de elefante" en una tufa localizada a 20 millas $(32 \mathrm{~km})$ al este de Parras, por lo cual se asignó a esta formación una posible edad Pleistoceno. Posteriormente (Rogers et al., 1961), con base en la presencia de un molar clasificado como Equus excelsus Leidy (1869), localizado a $7 \mathrm{~km}$ (4.3 millas) al nor-noroeste de Parras, se asignó una edad Pleistoceno para esta formación y se estimó un espesor de 100 a 200 m sin embargo, Ledezma-Guerrero (1967) propuso para la Formación Mayrán un espesor de 30 a 60 m. La Formación Mayrán fue dividida en dos partes (Wenzens, 1974 y Tardy, 1980): un cuerpo superior formado por estratos de travertino $\mathrm{y}$ caliche en la que reconocen numerosos miembros que se repiten, sobrepuestos a un cuerpo calcáreo inferior y más antiguo, siendo ambos cuerpos de edad Pleistoceno con fundamento en su posición estratigráfica y con apoyo de los datos paleontológicos anteriormente citados. Wenzens
(1974) consideró que la repetición de miembros en el cuerpo superior se debió a una alternancia de cambios climáticos entre períodos interglaciales de humedad y sequía.

Amezcua-Torres et al., (2012, Fig. 6) concentraron su trabajo en describir el cuerpo calcáreo inferior de la Formación Mayrán, constituido por una sucesión cíclica de cuerpos de caliza lacustre que terminan con paleosuelos y evaporita; estas capas se interdigitan lateral y verticalmente con rocas siliciclásticas de granulometría variable y al conjunto le asignaron espesores de 6 a 20 metros depositados en subcuencas escalonadas, conectadas entre sí por puntos de derrame (spill point) formados por tufa, y propusieron una edad isotópica de 3.9 Ma para el cuerpo inferior de la Formación Mayrán. En su descripción (Amezcua-Torres et al., 2012) solamente consideraron al cuerpo inferior como Formación Mayrán, y segregaron a las tufas y travertinos del cuerpo superior como depósitos post-Mayrán (citados por Wenzens, 1974 y Tardy, 1980 e incluidos en la Formación Mayrán originalmente definida por Imlay, 1936).

Es importante citar que Imlay (1936) y seguidores separaron a los suelos, no incluyéndolos en la definición de la Formación Mayrán y que la edad de estos suelos reviste interés en el contexto estratigráfico del área. Butzer et al., (2008) realizaron el estudio geomorfológico de capas de suelos en una amplia región en Coahuila y Durango y apoyados con isotopía estimaron que los suelos más antiguos en el área de Parras tienen edades entre 9400 a 7200 años (Holoceno), separados de suelos más jóvenes por un hiato de 500 años de duración.

Uno de los objetivos del presente artículo consiste en la redefinición litoestratigráfica de la Formación Mayrán, proponiéndola ahora como una aloformación, justificando este cambio por la presencia de discordancias internas en

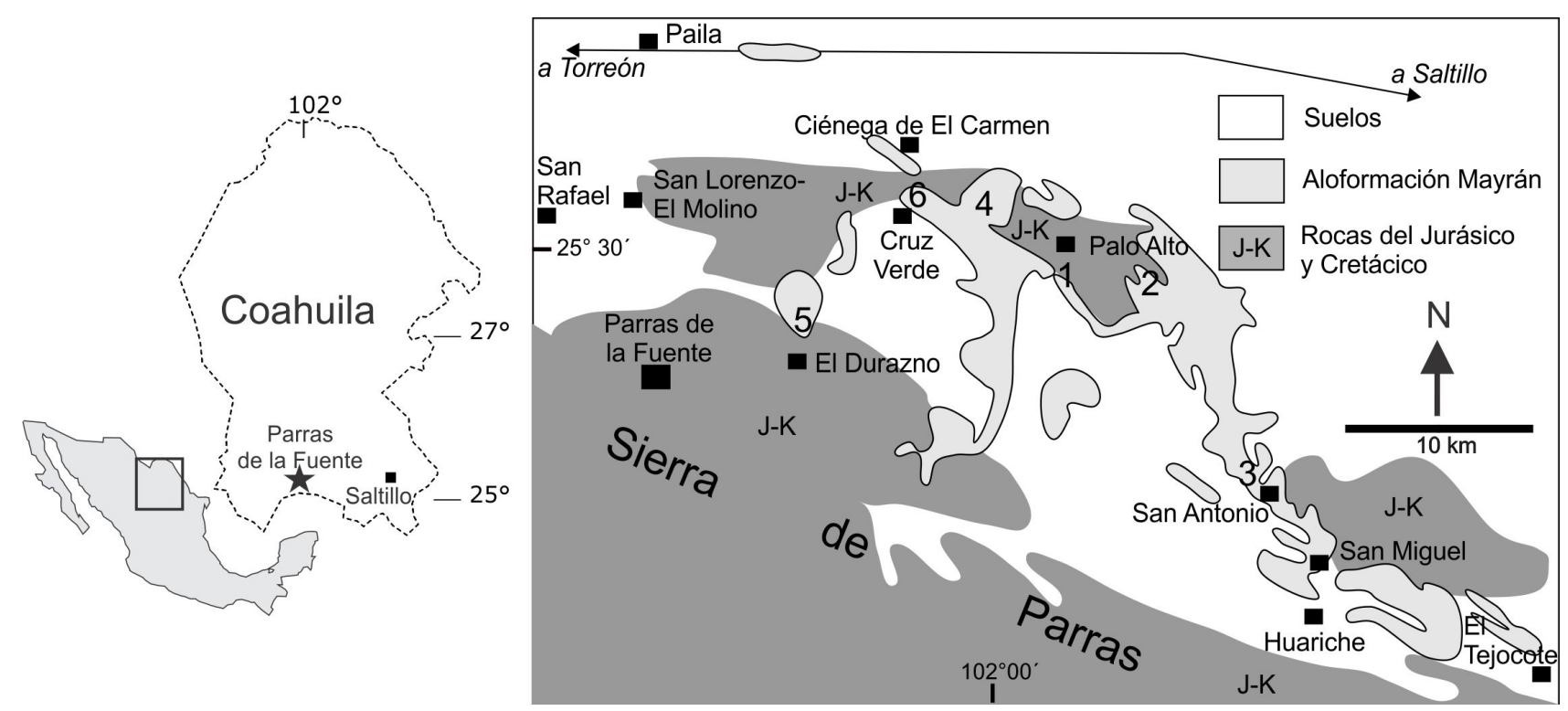

Figura 1. Localización del área de estudio. Lugares citados en el texto: 1) sur del poblado Palo Alto, 2) cantera de Palo Alto, en la mesa del Gato, 3) mesa de San Antonio, 4) mesa Los Bueyes, 5) mesa El Durazno y 6) mesa Cruz Verde. 
capas de carbonato y terrígenos con diferente edad, y así, no excluir al cuerpo superior dejándolo sin una nomenclatura apropiada. Otro propósito es describir por primera vez las características morfológicas de los molares de Equus sp., encontrados en este trabajo, en el cuerpo superior de los carbonatos Mayrán y que sustentan asignar una edad Pleistoceno, mientras que el cuerpo inferior de carbonatos Mayrán, por isotopía (3.9 Ma) puede corresponder al Plioceno (Amezcua-Torres, 2012). Ambos propósitos son importantes para tener un orden litoestratigráfico que apoye estudios futuros sobre paleo-ambiente, paleo-ecología y otros trabajos adicionales del Neógeno.

\section{Descripción litoestratigráfica}

Los mejores afloramientos de las capas Mayrán están al sur del poblado de Palo Alto, en la cantera de Palo Alto situada sobre la mesa del Gato (Figura 2), mesa de San Antonio, mesa de los Bueyes, mesa El Durazno y en la mesa de Cruz Verde (Tabla 1). La descripción litoestratigráfica

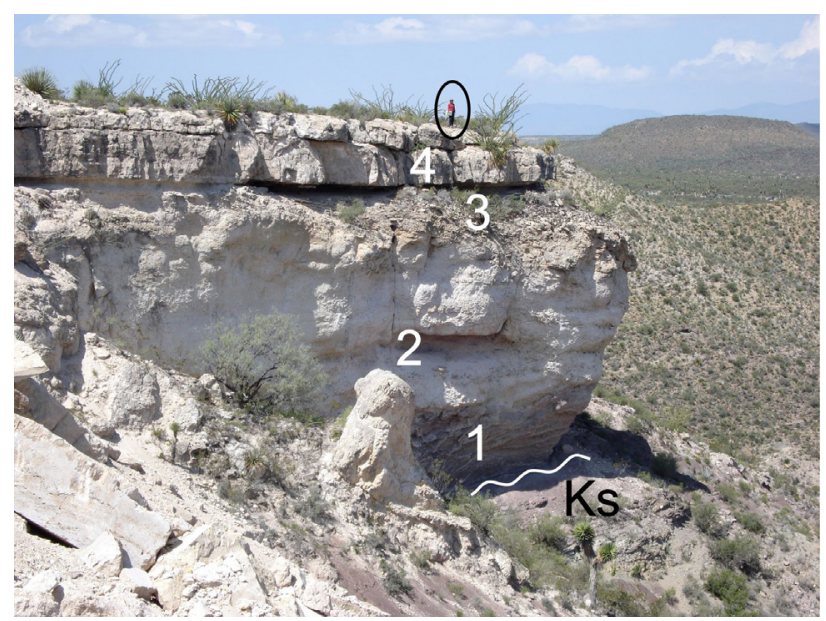

Figura 2. Aloformación Mayrán en la cantera de Palo Alto. Las unidades 1-4 corresponden al cuerpo inferior de edad Plioceno descrito en el texto y representado en la sección de consenso de los seis afloramientos estudiados en la Figura 3. Las capas inferiores Mayrán sobreyacen en discordancia angular a estratos del Grupo Difunta del Cretácico Superior (Ks). Escala mostrada en el círculo: $1.60 \mathrm{~m}$ de altura. detallada de estas localidades ha sido realizada por Amezcua -Torres (2012) y Amezcua-Torres et al., (2012, p. 977981) y no se considera necesario repetir su descripción y correlaciones estratigráficas en este artículo. La integración de los datos realizada en este trabajo muestra que las capas Mayrán se presentan en estratos horizontales, no deformados, que sobreyacen en discordancia angular a capas plegadas de arenisca, limolita y lutita, pertenecientes al Grupo Difunta del Cretácico Superior. Los estratos Mayrán en la cantera de Palo Alto (Figuras 2 y 3) son representativos del cuerpo inferior de todas las localidades arriba citadas y están compuestos por cuatro unidades. La unidad A1, en su base, tiene brecha de clastos de arenisca que provienen de rocas del Grupo Difunta, de 1 a $5 \mathrm{~cm}$ de diámetro, soportados por arcosa de grano medio a grueso en colores verde y ocre, en estratos laminares paralelos. El resto de esta unidad es arcosa conglomerática de la misma coloración con un espesor de 4.5 metros y la cima tiene una superficie irregular y un estrato de suelo calichoso de 10 a $15 \mathrm{~cm}$. La unidad A2 corresponde a otro nivel de arcosa de grano grueso y conglomerática de color blanquecino, con surcos y canales rellenos por conglomerado de caliza y arenisca. Dentro de esta unidad aparece un paleosuelo ocre que cubre a una superficie irregular seguida por una sucesión de tufa que subyace a estratos delgados de caliza mudstone y wackestone, que se acuñan lateralmente. La unidad A2 tiene de 8 a 10 metros de espesor. La unidad B3 está formada por capas de limolita y arenisca de grano fino a medio, en color verde, con espesor de $1.5 \mathrm{~m}$ que subyacen a caliza wackestone, de color crema; ambas litologías tienen estratos de 30 a $60 \mathrm{~cm}$ de espesor. Esta unidad tiene 3.5 metros de espesor y presenta cambios laterales de esta litología. La unidad B4 es caliza wackestone de bioclastos, laminar, de color gris claro a crema, con capas de 30 a 40 $\mathrm{cm}$ de espesor y su espesor incompleto es de 4 metros. Cada una de las sucesiones está separada por discordancias que se identifican como superficies de erosión.

El cuerpo superior de las capas Mayrán tiene su mejor exposición en las cercanías del poblado de Palo Alto. Se presentan varios ciclos de depósito formados por tufa y caliza arcillosa; estos ciclos están separados por superficies de erosión, lo que sugiere sucesivos periodos de depósito separados por intervalos de erosión (Figura 3, unidades C,

Tabla 1. Coordenadas de ubicación de las localidades referidas en el texto y descritas con detalle por Amezcua-Torres (2012) y Amezcua-Torres et al., (2012)

\begin{tabular}{ccccc}
\hline \multirow{2}{*}{ Número } & Localidad & $\begin{array}{c}\text { Latitud } \\
\text { Norte }\end{array}$ & $\begin{array}{c}\text { Longitud } \\
\text { Oeste }\end{array}$ & $\begin{array}{c}\text { Secciones de Amezcua-Torres, } 2012 \\
\text { y Amezcua-Torres et al., } 2012 .\end{array}$ \\
\hline 1 & sur de Palo Alto & $25^{\circ} 30^{\prime} 08^{\prime \prime}$ & $101^{\circ} 56^{\prime} 58^{\prime \prime}$ & Post-Mayrán \\
2 & cantera de Palo Alto & $25^{\circ} 31^{\prime} 04^{\prime \prime}$ & $101^{\circ} 55^{\prime} 42^{\prime \prime}$ & N7C \\
3 & mesa de San Antonio & $25^{\circ} 25^{\prime} 25^{\prime \prime}$ & $101^{\circ} 52^{\prime} 09^{\prime \prime}$ & N2913 \\
4 & mesa de Los Bueyes & $25^{\circ} 33^{\prime} 21^{\prime \prime}$ & $101^{\circ} 58^{\prime} 34^{\prime \prime}$ & N18B, N19, N21B y N22 \\
5 & mesa El Durazno & $25^{\circ} 27^{\prime} 31^{\prime \prime}$ & $102^{\circ} 05^{\prime} 58^{\prime \prime}$ & N37 \\
6 & mesa Cruz Verde & $25^{\circ} 31^{\prime} 24^{\prime \prime}$ & $102^{\circ} 03^{\prime} 30^{\prime \prime}$ & N39 \\
\hline
\end{tabular}




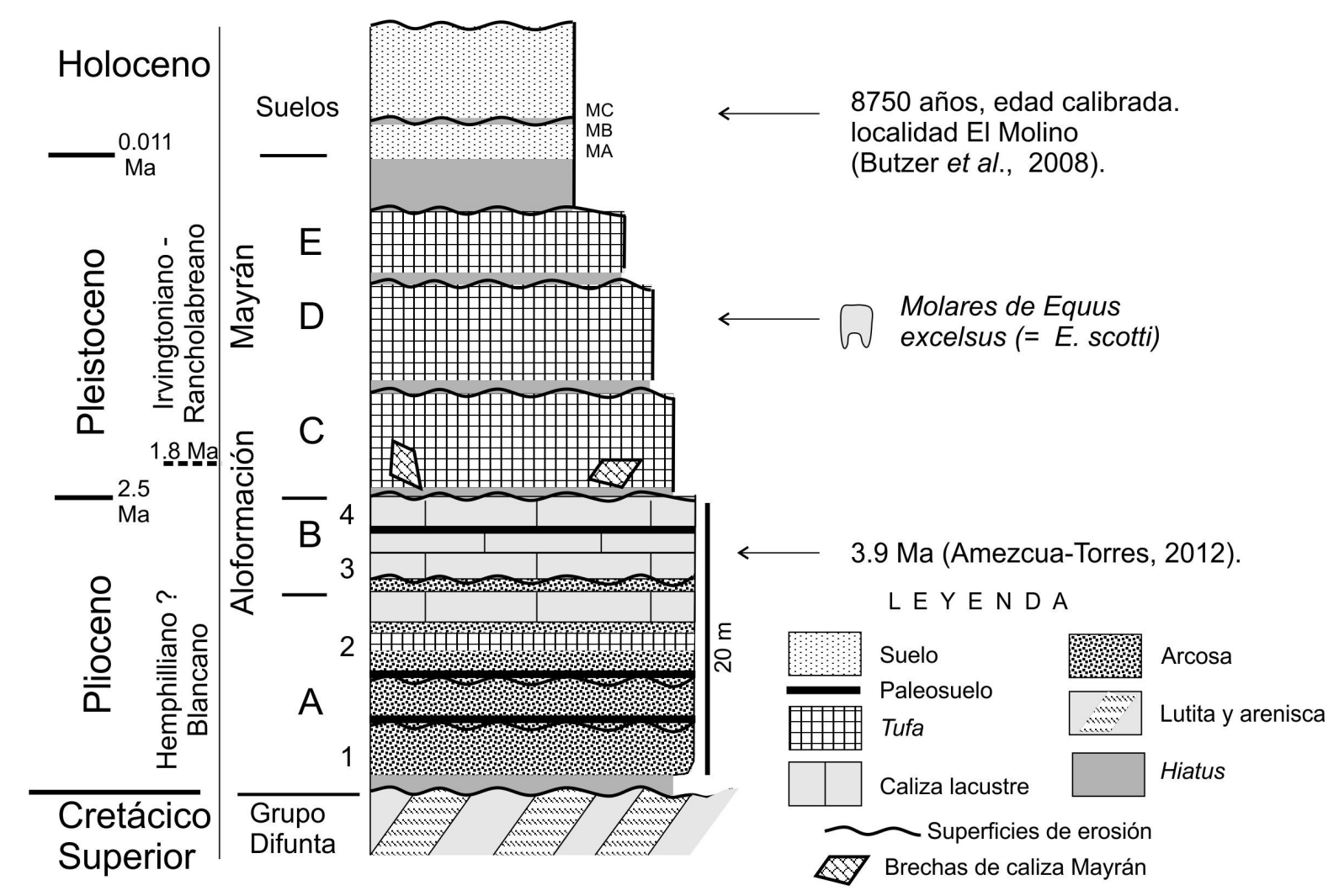

Figura 3. Sección estratigráfica compuesta de la Aloformación Mayrán. Las unidades se describen en el texto. Los cambios laterales de relieve representan perfiles de erosión relativos. Pisos y edades (Ma) en millones de años (North American Land Mammal Age NALMA e International Commission on Stratigraphy, 2012).

D y E). A estas capas, Amezcua-Torres et al., (2012) las denominaron como depósitos post-Mayrán, pero en este trabajo se considera más apropiado incluirlas como capas o miembro superior Mayrán. Su litología consiste de tufas de color café claro, intercaladas con caliza arcillosa, en estratos seudo-masivos, con espesor variable que puede alcanzar de 5 a 20 metros (Figura 4), con acuñamiento lateral. Estos depósitos se encuentran dispuestos en terrazas con niveles topográficos escalonados que separan a los niveles de tufa-carbonatos entre sí (Figura 4). En estas tufas (unidad C) existen clastos de caliza de unidades previamente depositadas de la Caliza Mayrán. La localidad tipo y sección estratigráfica representativa de estas sucesiones se localiza en las inmediaciones del poblado de Palo Alto. En uno de estos ciclos (unidad D) se encontraron tres molares de Equus sp. (Figura 5a-5c), en una cantera inmediata al sur del poblado de Palo Alto, municipio de Parras de la Fuente (Tabla 1).

En numerosos lugares se encuentran restos de vegetación fósil, tallos y hojas bien preservados, que muestran acumulaciones de plantas con variedad de formas, distribuidas tanto en las calizas de ambiente lacustre, en las tufas de los puntos de derrame entre las subcuencas lacustres y en las tufas más jóvenes del cuerpo superior de las capas Mayrán.
Los sedimentos más recientes en el área son suelos (Figura 6) conocidos como depósitos de malpaís, con un espesor variable, cubren en discordancia erosiva a las capas anteriormente descritas y se encuentran en la parte topográfica más baja. Butzer et al. (2008) determinaron en la base del suelo MC una edad calibrada de 8750 años mediante isótopos de $\mathrm{C}^{14}$, mientras que a las capas de suelo inferiores, denominadas MA y MB que son más antiguas, les dieron una edad máxima de 9400 años (Figura 3). En la localidad de El Durazno hay fósiles recolectados que tienen una edad de 11700 años, mientras que en San Juan de Vaquerías, Coahuila, su edad es de 41000 años, ambos casos datados con isotopía de $\mathrm{C}^{14}$ (Delgado, 2009).

\section{Discusión}

En las capas Mayrán se presentan varias litologías dependiendo de dos factores: uno es su proximidad con la fuente de aporte clástica y el otro con la producción de caliza o tufa. Se reconoce una sucesión estratigráfica inferior y más antigua, que tiene continuidad lateral y vertical en cada una de las subcuencas escalonadas de su depósito, con extensión en toda el área, pero con cambios laterales de litología entre carbonatos y rocas siliciclásticas, con espesor de 6 a $20 \mathrm{~m}$ 


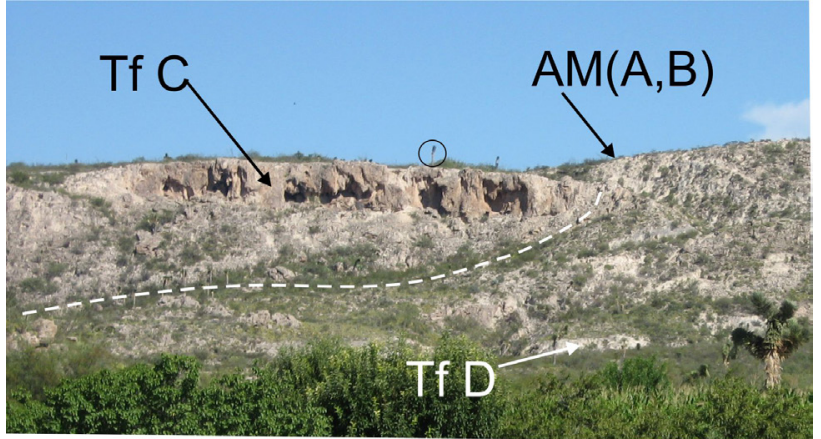

Figura 4. Localidad al sur del poblado de Palo Alto. Relaciones estratigráficas entre las unidades Mayrán: Tufas de las capas superiores Mayrán (Tf C y Tf D) y las capas inferiores de caliza lacustre Mayrán $\mathrm{AM}(\mathrm{A}, \mathrm{B})$. La línea blanca representa el contacto estratigráfico marcado por la superficie de erosión entre la tufa $\mathrm{Tf} \mathrm{C}$ y el cuerpo inferior de la Aloformación Mayrán AM(A-B). En la imagen se muestran las relaciones topográficamente escalonadas entre el cuerpo más antiguo AM(A-B), que subyace en discordancia erosiva al depósito $\mathrm{Tf} C$, el que a su vez es erosionado (no observado en la imagen) y subyace a las capas Tf D, que contiene molares de Equus sp. La escala se muestra en el círculo con una palma de aproximadamente $4 \mathrm{~m}$ de altura.

(Amezcua-Torres et al., 2012). Datos preliminares indican que la edad isotópica más antigua compuesta por capas bipartitas de carbonatos y rocas siliciclásticas, puede ser de 3.9 Ma (Amezcua-Torres, 2012), mientras que en carbonatos de punto de derrame (spill point) es de $2.5 \mathrm{Ma}$, siendo la primera cifra absoluta la edad más probable para el depósito más antiguo que corresponde con una muestra tomada en la caliza lacustre Mayrán. Esta caliza lacustre es una secuencia sedimentaria, separada en su base por una discordancia angular, que se encuentra sobre capas del Grupo Difunta, que con evidencia del presente estudio contienen Exogyra ponderosa, del Cretácico Superior, mientras que en su cima hay otra discordancia paralela, que la separa de masas de tufa, estratigráficamente más jóvenes, que contiene los restos molares de Equus sp.

La sucesión estratigráfica más joven, que Imlay (1936) incluyó en la definición original de la Formación Mayrán, consiste de cuerpos discontinuos de tufa, en acomodo topográfico escalonado (Figura 4). En esta sucesión superior se reconocen al menos dos depósitos de tufas y un espesor insignificante de carbonato impuro (Figura 3) en capas paralelamente horizontales y separadas entre sí por superficies de erosión, dispuestos topográficamente en forma escalonada y restringidos entre las subcuencas lacustres.

Por las características litológicas descritas para la Formación Mayrán y con base en los Artículos 7, 30, 58, 59 y 60, del Código Estratigráfico Norteamericano (Barragán et al., 2010), se estima conveniente redefinir formalmente a esta unidad litoestratigráfica como una aloformación. La Aloformación Mayrán es una secuencia sedimentaria cartografiable en la escala del área (Amezcua-Torres, 2012 y Amezcua-Torres et al., 2012), que se identifica por sus discontinuidades limitantes reconocibles lateralmente, con

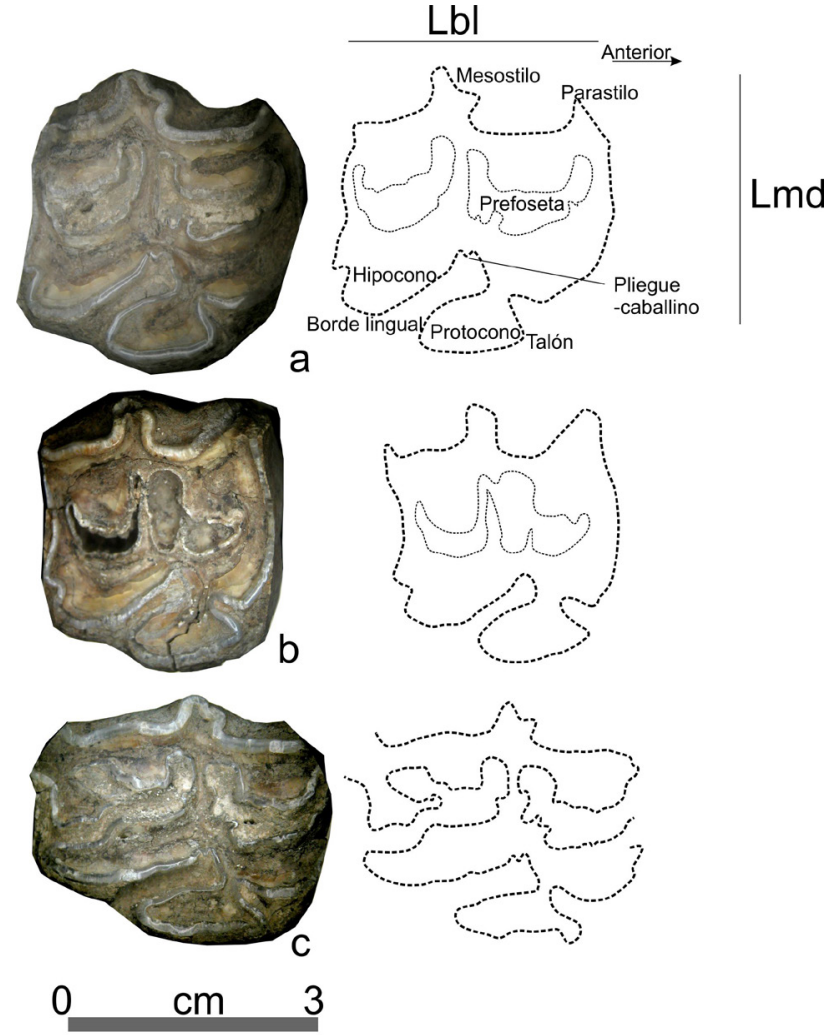

Figura 5. Molares: P4 derecho (a), M 1 (b) y M 3 (c) descritos en el texto. Lmd = Longitud mesio-distal, $\mathrm{Lbl}=$ Longitud buco-lingual. Escala gráfica para los tres ejemplares.

variaciones físicas de litología y edad, tiene varios cuerpos de rocas carbonatadas y siliciclásticas depositadas en ambiente lacustre, su contacto inferior está en discordancia angular sobreyaciendo a rocas del Cretácico Superior y su contacto superior subyace a suelos del Holoceno. Su localidad tipo y estratotipo (Figura 3) se encuentra al sur del poblado de Palo Alto y las secciones de referencia en las localidades de cantera Palo Alto, mesas de San Antonio, Los Bueyes, Cruz Verde y El Durazno (Tabla 1).

La edad de la Aloformación Mayrán varía del Plioceno al Pleistoceno. Originalmente estas capas fueron asignadas al Pleistoceno (Rogers et al., 1961) por la presencia de Equus excelsus Leidy (1869), lo cual es controversial porque no se sustentó su determinación con evidencia taxonómica. En este trabajo se reporta el hallazgo de tres molares de Equus sp., ubicados en la unidad D del cuerpo superior Mayrán. El material fósil colectado (Figura $5 \mathrm{a}-\mathrm{c}$ ) consiste de tres molares superiores, que se presume corresponden al mismo individuo. El desgaste que se observa en los molares, sugiere que los especímenes pertenecen a un individuo adulto viejo. Los ejemplares son un premolar derecho $\mathrm{P} 4$ (Figura 5 a), que es el mejor conservado; un molar M1 (Figura $5 \mathrm{~b}$ ), con la superficie oclusal muy desgastada, especialmente la prefoseta y un molar M3 (Figura $5 \mathrm{c}$ ) bien conservado. El P4, tiene la superficie oclusal bien conservada, el mesostilo y parastilo están bien desarrollados, 


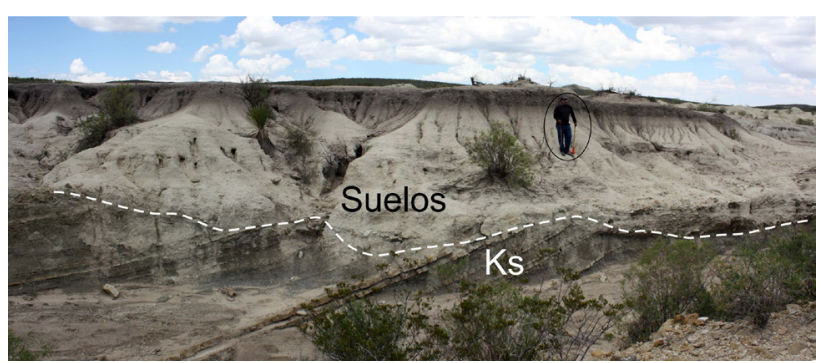

Figura 6. Suelos no consolidados de edad Holoceno, en discordancia indicada por la línea blanca cortada, sobre capas plegadas del Grupo Difunta del Cretácico Superior (Ks). Escala gráfica en el círculo: 1.70 $\mathrm{m}$ de altura.

el protocono es sub-redondeado a sub-triangular, ancho, con el borde lingual recto, el talón está muy reducido; el hipocono está desgastado, sólo queda un vestigio del esmalte, además presenta un pliegue caballino casi imperceptible (pli caballin). La pieza M3, por su posición anatómica, presenta gran variación en las estructuras de la superficie oclusal y en el molar M1 la superficie oclusal está parcialmente destruida, especialmente la prefoseta. El desgaste de los molares pudiera deberse a la dieta. En los especímenes con un desgaste equivalente, las estructuras de la superficie oclusal son similares, incluyendo la presencia de un pliegue caballino muy reducido en molares muy gastados, pero bien desarrollado en molares con menor desgaste. Los estilos parastilo y mesostilo son similares. El protocono es ancho con el talón muy reducido, el borde lingual siempre es recto, a diferencia de molares con menor desgaste cuyo borde lingual es cóncavo. El tamaño de los ejes mesio-distal y buco-lingual de los especímenes de Mayrán fueron comparados con molares referidos a Equus excelsus (= Equus scotti sensu Carranza-Castañeda y Roldán-Quintana, 2007), recolectados en río Vírgenes en el estado de Nuevo León (consignados en el Museo de Historia Natural del condado de Los Ángeles) y no existen diferencias significativas para diferenciarlos en estado de desgaste comparable. Mooser y Dalquest (1975), en su descripción de E. excelsus de la fauna de El Cedazo en el estado de Aguascalientes, definen caracteres de los molares superiores para reconocer a esta especie; entre los más constantes, a pesar del desgaste del molar, están los estilos bien definidos (mesostilo y parastilo), la superficie oclusal simple y protocono ancho, con el talón reducido y el borde lingual cóncavo; estos caracteres son similares en los molares de Mayrán.

La definición de E. excelsus originalmente propuesta por Leidy (1869) en Nebraska, fue considerada como una especie no bien definida por Gidley (1901) y renombrada por Villada (1903) como E. occidentalis y posteriormente como E. mexicanus por Hibbard (1955). Algunos autores (Ferrusquía-Villafranca, 1978; Kurten y Anderson, 1980) sugirieron que $E$. conversidens había sido la primera especie válida descrita para el Pleistoceno temprano de América del Norte.Reynoso-Rosales y Montellano-Ballesteros
(1994) abordaron el problema de la identificación impropia del género E. excelsus en México y consideraron que su determinación, en muchos casos es imprecisa, por lo que la especie descrita por Mooser y Dalquest (1975) era más conveniente reconsiderarla como $E$. conversidens y por lo tanto, otros elementos del cráneo y metapodiales es conveniente incluirlos para diagnosticar la identificación correcta de la especie. Actualmente se ha precisado que en México existieron cinco especies de caballos en el Pleistoceno: Equus tau (o E. francisci), E. conversidens, E. excelsus, E. niobrarensis y E. mexicanus (Bravo-Cuevas et al., 2011).

La presencia de E. excelsus (=E. scotti) se ha reportado en Sonora (White et al., 2010), Jalisco (Lucas, 2008), Hidalgo (Villarello y Böse, 1902) y recientemente en San Juan de La Vaquería, área de Saltillo (Arellano, 2011; Delgado, 2009), por lo tanto, E. excelsus tiene una distribución amplia. En general, la edad de los especímenes Equus varía del Pleistoceno al Reciente, pero con la presencia de Bison podría ubicarse en el Pleistoceno tardío (Rancholabreano, Montellano-Ballesteros, 1992). Sin esta asociación, E. excelsus es asignado sólo al Pleistoceno (Irvingtoniano-Rancholabreano de 2.5 Ma a 12000 años).

En conclusión, los especímenes de Mayrán preliminarmente son referidos a E. excelsus (=E. scotti), con base en la forma y tamaño del protocono y los estilos (mesostilo y parastilo bien desarrollados), sin embargo, se requieren otros elementos óseos y la asociación con otros mamíferos para tener mayor definición, por lo que es conveniente realizar estudios complementarios. Dos molares descritos en este trabajo se encuentran resguardados en la Colección Nacional de Paleontología, en el Instituto de Geología, de la Universidad Nacional Autónoma de México, Ciudad Universitaria México, D. F., números de catálogo IGM 2, IGM 6262 (Figura 5 a) e IGM 6263 (Figura 5 c).

El espesor máximo de carbonato lacustre en la secuencia inferior de la Formación Mayrán no excede de 20 m. Los datos obtenidos por Amezcua-Torres (2012) sugieren condiciones de alta a moderada productividad de carbonato derivado de abundante actividad orgánica. Asumiendo una tasa de sedimentación de carbonato mínima de $1 \mathrm{~mm} /$ año (Brunskill, 1969; Müller y Suess, 1979; Strasser y Samankassou, 2003), en condiciones lacustres normales, con un clima templado y una compactación reducida, dicho espesor pudiera haberse acumulado en 80000 años aproximadamente, sin tomar en cuenta las interrupciones ocurridas en la sucesión cíclica de su depósito. Esto nos lleva a considerar que la edad absoluta de 3.9 Ma y su relación con la tasa de acumulación del sedimento, el depósito del cuerpo inferior no podría ser más joven a 3.0 Ma. Por lo tanto, la secuencia inferior (calizas y rocas siliciclásticas) y la secuencia superior de tufas (excluidas las tufas de los puntos de derrame) de los estratos Mayrán, poseen edades diferentes: la inferior, Plioceno y la superior, Pleistoceno, separadas por períodos de erosión con interrupciones prolongadas en su depósito. 
Es conveniente mencionar que en las tufas de la secuencia superior y en los puntos de derrame de la secuencia inferior de la Formación Mayrán, existen numerosos moldes de plantas, y en ambas secuencias se reconocen elementos foliares y tallos. La morfología foliar se presenta en dos tipos principales; lanceoladas con ápice agudo, angostas y alargadas con margen dentado regular (Figura 7 a), así como formas semicirculares amplias, de tipo palmado-lobada, con base aparentemente cordada (Figura 7 b). La densidad de tallos y hojas con diversas formas sugieren condiciones propicias de humedad suficiente para proliferar y acumularse. Un estudio morfológico detallado de este conjunto foliar ayudará a una mejor estimación de las condiciones paleo-climáticas que prevalecieron en estas latitudes para este tiempo. Aguilar y Velasco-de León (2002) en la región de Santa María Amajac, describen la morfología foliar de la vegetación que prevaleció en el Plioceno de esa área, e infieren que esa vegetación posiblemente existió en condiciones climáticas templadas a sub-húmedas, con precipitación media anual estimada en $650 \pm 120 \mathrm{~mm}$ (García, 1973), que corresponde a una temperatura media anual para la República Mexicana de entre $12^{\circ}$ a $18^{\circ} \mathrm{C}$,

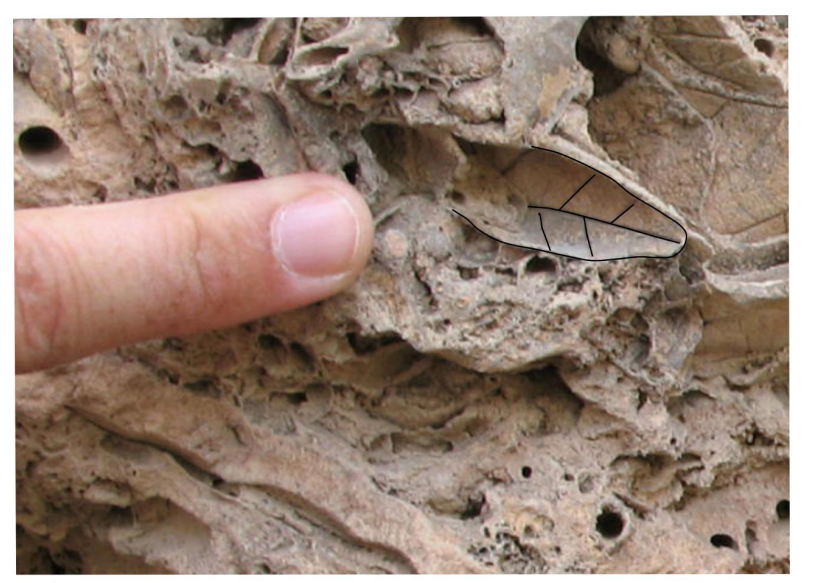

a)
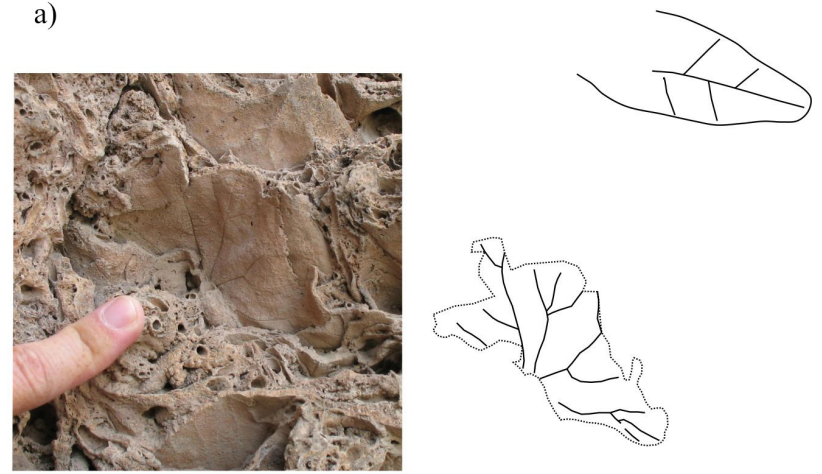

b)

Figura 7. Restos foliares fósiles con diferente morfología: a) lanceolada de ápice agudo y borde dentado con espaciamiento regular, observada en la tufa entre Palo Alto y la mesa Los Bueyes; b) formas semicirculares amplias, de tipo palmado-lobada, con base aparentemente cordada, observada en la tufa ciénega de El Carmen, al norte-poniente de la mesa Los Bueyes. aclarando que Santa María Amajac se encuentra en una latitud más austral que la localidad de Parras de la Fuente.

Es de hacer notar que en Santa María Amajac, Hidalgo (Beltrán-Romero y Luna-Gómez, 1994); en Parras de la Fuente, Coahuila (Amezcua-Torres et al., 2012), y en la Formación Providencia en Cruillas, Tamaulipas (Rodríguez y Werner, 1993), en la base de estas sucesiones estratigráficas, se encuentran capas de carbonatos y restos vegetales del Neógeno tardío; esta interrelación requiere de un análisis sedimentológico más detallado para deducir las paleo-temperaturas y los paleo-climas regionales.

Las capas de la Aloformación Mayrán se explotan como canteras para materiales de construcción y su calidad depende de las facies de depósito, por lo que su estudio reviste interés económico. Otro interés que tienen estas capas consiste en su espectacular preservación, como depósito lacustre sin deformación, ejemplo análogo para el estudio de cuencas petroleras, y además un sitio natural que puede ser propuesto como un geoparque con reconocimiento de la UNESCO (Organización Educativa, Científica y Cultural de las Naciones Unidas, por sus siglas en inglés).

\section{Conclusiones}

Las capas Mayrán están formadas por rocas siliciclásticas, caliza y tufa, que forman sucesiones de ciclos, pero separados por interrupciones en su depósito que se identifican como discordancias paralelas. La base de estas capas descansa en discordancia angular sobre estratos plegados, que contienen fósiles del Cretácico Superior, mientras que su cima subyace con discordancia erosiva a suelos del Holoceno. Las capas de la Aloformación Mayrán no están deformadas. Según los datos isotópicos (3.9 Ma) reportados por Amezcua-Torres (2012) para el cuerpo inferior Mayrán, compuesto por caliza y rocas siliclásticas, corresponde una edad Plioceno, mientras que las tufas del cuerpo superior (excluidas las tufas de puntos de derrame), con presencia de vertebrados preliminarmente identificados como Equus excelsus (=E. scotti), son del Pleistoceno. Por las características descritas en este trabajo, nosotros proponemos considerar como aloformación a todos los depósitos Mayrán, mismos que Imlay (1936) denominó como Formación Mayrán.

\section{Agradecimientos}

Se agradece al Dr. Ismael Ferrusquía-Villafranca por sus observaciones preliminares sobre un molar estudiado. A la Dra. Marisol Montellano-Ballesteros y al Mtro. José M. Padilla-Gutiérrez, por su orientación referente al género Equus. A Claudio Bartolini por la revisión del resumen de este artículo. Muy especial agradecimiento por sus valiosos y adecuados comentarios a los revisores: Dra. María Teresa Alberdi, Dr. Javier Helenes-Escamilla y dos revisores anónimos. Al cuerpo editorial del Boletín, Dr. 
Antoni Camprubí Dr. Juan Carlos García Barragán y Denise Hernández Villalva, por sus acertados comentarios, esmero y apoyo para mejorar las ideas aquí expresadas. Natalia Amezcua por sus observaciones litoestratigráficas.

\section{Referencias}

Aguilar, F.J., Velasco-de León, M.P., 2002, El clima durante el Plioceno en la región de Santa María Amajac, Hidalgo, México: Boletín de la Asociación Botánica, México, 71, 71-81.

Amezcua-Torres, N., 2012, Stratigraphy and facies of the Pliocene Mayran lacustrine basin system: Manchester, United Kingdom, University of Manchester, tesis doctoral, $205 \mathrm{p}$.

Amezcua-Torres, N., Gowtorpe, R., MacQuaker, J., 2012, Cascading carbonate lakes of the Mayrán basin system, northeast of Mexico: The interplay of inherited structural geometry, bed rock lithology, and climate: Geological Society Bulletin, 124, (5/6), 975-988.

Arellano, T.Z., 2011, Los mejores fósiles de Coahuila, La herencia paleontológica de Coahuila tiene muchos capítulos nuevos que contar, disponible en: www.astronomos.org/articulistas, consultado 11 de enero de 2013.

Barragán, R., Campos-Madrigal, E., Ferrusquía-Villafranca, I., López-Palomino, I, Tolson, G., 2010, Código Estratigráfico Norteamericano: Universidad Nacional Autónoma de México, Instituto de Geología, Boletín, 117, 47 p.

Beltrán-Romero, F., Luna-Gómez, P., 1994, Estudio geológico de la región de Santa María Amajac, Municipio de Atotonilco el Grande, Estado de Hidalgo: México, Distrito Federal, Universidad Nacional Autónoma de México, tesis de licenciatura, $131 \mathrm{p}$.

Bravo-Cuevas, V., Jiménez-Hidalgo, E., y Priego-Vargas, J., 2011, Taxonomía y hábito alimentario de Equus conversidens (Perissodactyla, Equidae) del Pleistoceno Tardío (Rancholabreano) de Hidalgo, Centro de México: Revista Mexicana de Ciencias Geológicas, 28, (1), 65-82.

Brunskill, G.J., 1969, Fayetteville Green Lake, New York, II: Precipitation and sedimentation of calcite in a meromictic lake with laminated sediments: Limnology and Oceanography, 14, (6), 830.

Butzer, K.W., Abbott, J.T., Frederick, C.D., Lehman, P.H., Cordova, C.E., Oswald, J.F., 2008, Soil-geomorphology and "wet" cycles in the Holocene record of North-Central Mexico: Geomorphology, 101, 237-267.

Carranza-Castañeda, O., Roldán-Quintana, J., 2007, Mastofauna de la Cuenca de Moctezuma, Cenozoico tardío de Sonora, México: Revista Mexicana de Ciencias Geológicas, 24(1), 81-88.

Delgado, R.C., 2009, Edad del hielo en Coahuila, VANGUARDIA, disponible en $<\mathrm{http} / / / \mathrm{www}$.vanguardia.com.mx/edad_de_hielo en_coahuila-439434.html>, publicado sábado, 05 de diciembre del 2009 , fecha de consulta 11 enero 2013.

Ferrusquía-Villafranca, I., 1978, Distribution of Cenozoic vertebrate faunas in Middle America and problems of migration between North and South America, en Ferrusquía-Villafranca, I. (ed.), Conexiones terrestres entre Norte y Sudamérica: Universidad Nacional Autónoma de México, Instituto de Geología, Boletín 101, 193-321.

García, E., 1973. Modificación al sistema de clasificación climática de Köppen (Adaptado para la República Mexicana): México, Distrito Federal, Universidad Nacional Autónoma de México, Instituto de Geografía, $246 \mathrm{p}$.

Gidley, J.W., 1901, Tooth characters and revisions of the North American species of the genus Equus: American Museum of Natural History Bulletin, 14, 91-142.

Hibbard, C.W., 1955, Pleistocene vertebrates from the Upper Becerra (Becerra Superior) Formation, valley of Tequisquiac, Mexico, with notes on other Pleistocene forms: University of Michigan, Museum of Palaeontology Contributions, 12, 47-96.

Imlay, R.W., 1936, Evolution of the Coahuila Peninsula, Mexico, Part IV: Geology of the western part of the Sierra de Parras: Geological Society of America Bulletin, 47, 1091-1152.
International Commission on Stratigraphy, 2012, International Chronostratigraphic Chart, disponible en $<$ http://www.stratigraphy. org/ICSchart/ChronostratChart2013-01.pdf>, página consultada el 15 de febrero del 2013.

Kurten, B., Anderson, E., 1980, Pleistocene Mammals of North America: New York, United States of America, Columbia University Press, $442 \mathrm{p}$.

Ledezma-Guerrero, O., 1967, Resumen de la Geología de la hoja Parras, Estados de Coahuila, Durango y Zacatecas: Universidad Nacional Autónoma de México, Instituto de Geología, Carta Geológica de México, hoja Parras 13R-1(6), serie 1:100000.

Leidy, J., 1869, On the extinct mammalian of Dakota and Nebraska: Journal of the Academy of Natural Sciences of Philadelphia, 2, 257- 401.

Lucas, S.G., 2008, Late Cenozoic fossil mammals from the Chapala rift basin, Jalisco, Mexico, en Lucas, S.G., Morgan, G.S., Spielmann, J.A., Prothero, D.R. (eds.), Neogene mammals: New Mexico, United States of America, New Mexico Museum of Natural History and Science Bulletin, 44, 39-49.

Montellano-Ballesteros, M., 1992, Una edad del Irvingtoniano al Rancholabreano para la fauna Cedazo del Estado de Aguascalientes: Universidad Nacional Autónoma de México, Instituto de Geología, Revista, 9 (2), 195-203.

Mooser, O., Dalquest, W., 1975, Pleistocene mammals from Aguascalientes, central Mexico: Journal of Mamalogy, 56, 781-820.

Müller, P.J., Suess, E., 1979, Productivity, sedimentation rate, and sedimentary organic matter in the oceans-I: Organic carbon preservation: Elsevier, Deep Sea Research Part A., Oceanographic Research Papers, 26 (12), 1347-1362.

Reynoso-Rosales, V.H., Montellano-Ballesteros, M., 1994, Revisión de los équidos de la fauna Cedazo del Pleistoceno de Aguascalientes, México: Revista Mexicana de Ciencias Geológicas, 11 (1), 87-105.

Rodríguez, F., Werner, J., 1993, Investigación preliminar de la Formación Providencia (calizas terciarias de agua dulce) al este de Linares, N. L., México: Actas de la Facultad de Ciencias de la Tierra, Universidad Autónoma de Nuevo León, 8p.

Rogers, C.L.; Cserna, Z. de, Vloten, R. van, Tavera-Amezcua, E., OjedaRivera, J., 1961, Reconocimiento geológico y depósitos de fosfatos del norte de Zacatecas y áreas adyacentes en Coahuila, Nuevo León y San Luis Potosí: Consejo de Recursos Naturales no Renovables, Boletín 56, $322 \mathrm{p}$.

Strasser, A., Samankassou, E., 2003, Carbonate sedimentation rates today and the past: Holocene of Florida Bay, Bahamas, and Bermuda vs. Upper Jurassic and Lower Cretaceous of the Jura Mountains (Switzerland and France): Geologia Croatica, 56 (1), 1-18.

Tardy, M., 1980, Contribution à l'étude géologique de la Sierra Madre Orientale du Mexique: Paris, France, Université Pierre et Marie Curie, tesis doctoral, $459 \mathrm{p}$.

Villada, M., 1903, Apuntes acerca de la fauna fósil del valle de México: México, Distrito Federal, Museo Nacional de México, Anales, $1^{\mathrm{a}}$. Época, 7, 441-451.

Villarelo, J. y Böse, E. 1902, Criaderos de Fierro de la Hacienda de Vaquerías en el Estado de Hidalgo: Boletín del Instituto Geológico Mexicano, 16, 15-44.

Wenzens, G., 1974, Morphologische entwicklung ausewählter regionen nordmexikos, unter besonderer berücksichtigung des kalkkrusten, piedement- und poljeproblems: Düsseldorfer Geographische Schriften, Heft 2, $330 \mathrm{p}$.

White, R.S., Mead, J.I., Baez, A., Swift, S.L., 2010, Localidades de vertebrados fósiles del Neógeno (Mioceno, Plioceno y Pleistoceno): una evaluación preliminar de la biodiversidad del pasado, en MolinaFreaner, F.E., Van Devender, T.R., (eds.), Diversidad biológica de Sonora: Universidad Nacional Autónoma de México y Comisión Nacional para el Conocimiento y Uso de la Biodiversidad, México, 51-72.

Manuscrito recibido: Agosto 20, 2012.

Manuscrito corregido recibido: Febrero 20, 2013.

Manuscrito aceptado: Marzo 18, 2013. 